VOL. $24(1981),|-1|$.

\title{
MANIFOLDS OF SMOOTH MAPS
}

\author{
Truong Công Nghê
}

\begin{abstract}
We show that the space of smooth maps from a compact smooth manifold into another smooth manifold can be endowed with the structure of a smooth manifold if we use the $\Gamma$-differentiation of Yamamuro. We then generalise the Smale Density Theorem to mappings between these manifolds.
\end{abstract}

The main purpose of this paper is to show that the space $C^{\infty}\left(Y_{1}, Y\right)$ of smooth maps from a compact $C^{\infty}$ manifold $X$ into a finite-dimensional $C^{\infty}$ manifold $Y$ can be endowed with the structure of a smooth manifold if we use the T-differentiation of Yamamuro [12]. Here, for simplicity, we suppose that $y$ is finite-dimensional even though the result can be easily extended to an infinite-dimensional $Y$ admitting a smooth spray [1]. Similar results have been obtained by Leslie [8] and Gutknecht [4] using the $C_{\pi}^{\infty}$ differentiability of Keller and the $C_{\Gamma}^{\infty}$ differentiability of Fischer respectively [3], [7]. The case of a non-compact $X$ has also been investigated by Michor [9] using Keller's $C_{\pi}^{\infty}$ differentiability.

The paper consists of three sections. In $\S 1$, we define general manifolds modelled on a $\Gamma$-family [12] of locally convex spaces, manifolds which we term as $\Gamma$-manifolds. Then corresponding to the $B \Gamma$-differentiability in [12]'we have the subclass of $B \Gamma$-manifolds. Section 2 is for showing that $C^{\infty}(X, Y)$ is in general a $\Gamma$-manifold of class $C^{\infty}$. We also give examples of $B \Gamma$-manifolds of class $C^{\infty}$. The last section, $\$ 3$, is devoted to a generalisation of the Smale Density

Received 9 January 1981. The author wishes to thank S. Yamamuro for his supervision as well as his many constructive discussions. 
Theorem [2] to the case of $B \Gamma$-maps between $\Gamma$-manifolds.

This paper depends heavily on [12] and some of its results have been announced in [10] and [11].

\section{1. $\Gamma-$ and $B \Gamma-$ manifolds}

Let $F$ be a $\Gamma$-family of locally convex spaces [12] and $X$ be a Hausdorff space. A $\Gamma$-chart on $X$ is a triple $(U, \alpha, E)$ of an open set $U$ of $X, E \in F$ and a homeomorphism $\alpha$ of $U$ into $E$. Let $k \geq 1$ be an integer. Then, two $\Gamma$-charts $(U, \alpha, E)$ and $(V, \beta, F)$ are said to be $C_{\Gamma}^{k}$-compatible if the transition map $\beta \circ \alpha^{-1}: \alpha(U \cap V) \rightarrow \beta(U \cap V)$ is a $c_{\Gamma^{k}}^{k}$ diffeomorphism [12].

A collection of $\Gamma$-charts $\left\{\left(U_{\alpha}, \alpha, E_{\alpha}\right)\right\}$ is called a $\Gamma$-atlas of class $c^{k}$ if

(1) $\left\{U_{\alpha}\right\}$ is a covering of $X$,

(2) each member is $c_{\Gamma}^{k}$-compatible with every member of the collection.

It is said to be maximal if every $\Gamma$-chart that is $c_{\Gamma}^{k}$-compatible with all members of the atlas belongs to the atlas.

A r-manifold of class $c^{k}$ modelled on the family $F$ is a Hausdorff space $X$ equipped with a maximal $\Gamma$-atlas of class $c^{k}$. Each $\Gamma$-chart in the maximal $\Gamma$-atlas will be called an admissible chart. A $\Gamma$-chart $(U, \alpha, E)$ is said to be (centred) at $x \in X$ if we have $x \in U$. If the model spaces coincide with a single $E \in F$ then the $\Gamma$-manifold is said to be modezzed on $E$. When the transition maps are $C_{B \Gamma^{-m a p s}}^{k}$ the $\Gamma$-manifold is called a $B \Gamma$-manifold.

Let $X$ and $Y$ be $\Gamma$-manifolds of class $c^{k}$ modelled on the same family $F$. A map $f: X \rightarrow Y$ is said to be of class $c_{\Gamma}^{k}$ or a $c_{\Gamma}^{k}$-map if, for each $x \in X$ and each admissible chart $(V, B, F)$ on $Y$ centred at 
$f(x)$, there exists an admissible chart $(U, \alpha, E)$ on $X$ centred at $x$, such that $f(U) \subseteq V$ and the Zocal representative of $f$, $\beta \circ f \circ \alpha^{-1}: \alpha(U) \rightarrow \beta(V)$, is a $C_{\Gamma^{-m a p}}^{k}$. In a similar manner, the $C_{B \Gamma^{-}}^{k}$ maps between $B \Gamma$-manifolds of class $C^{k}$ modelled on the same $\Gamma$-family can be defined.

Let $F$ be a $\Gamma$-family and $X$ be a $\Gamma$-manifold of class $C^{k}$ modelled on $F$. We shall always assume that $\mathbb{R} \in F$ and $\Gamma_{\mathbb{R}}$ consists of the absolute value norm. A $\Gamma$-curve at $x \in X$ is a $C_{\Gamma}^{\mathcal{l}}$-map $c$ from an open subset of $\mathbb{R}$ containing zero into $X$ such that $c(0)=x$. By definition, if $c$ is a $\Gamma$-curve at $x \in X$ and $(U, \alpha, E)$ is an admissible chart at $x, \alpha \circ c$ is a $C_{\Gamma}^{l}$-map into $E$. Since $L_{\Gamma}(\mathbb{R}, E)=E, \quad\left(\alpha^{\circ} c\right)^{\prime}(0)$ is identified with an element of $E$.

We define the $\Gamma$-tangent space $T_{x} X$ of $X$ at $x$ as the set of usual equivalent classes of $\Gamma$-curves at $x$ [2]. Then, every admissible chart $(U, \alpha, E)$ at $x$ defines a bijection of $T_{x} X$ onto $E$ and if $(V, B, F)$ is another admissible chart at $x, E$ and $F$ are $\Gamma$-isomorphic; they are $B \Gamma$-isomorphic when $X$ is a $B \Gamma$-manifold. By this bijection, we transplant the locally convex structure of $E$, including its calibration, onto $T_{x} X$. Therefore, the family $\left\{T_{x} X: x \in X\right\}$ becomes a $\Gamma$-family.

When $X$ and $Y$ are $\Gamma$-manifolds of class $c^{k}(k \geq 1)$ modelled on a family $F$ and $f: X+Y$ is a $C_{\Gamma}^{k}$-map, then we have the $\Gamma$-tangent map of $f: T_{x} f: T_{x} X \rightarrow T_{f(x)^{Y}}$. Similarly the $B \Gamma$-tangent map can be defined for

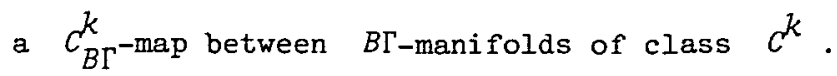

\section{The $\Gamma$-manifold $C^{\infty}(X, Y)$}

Let $X, Y$ be finite-dimensional $C^{\infty}$ manifolds without boundary, $X$ being compact. In order to show that $C^{\infty}(X, Y)$ is a $\Gamma$-manifold of class $C^{\infty}$, we first prove the I-version of the Omega Lemma [1, Corollary 3.8]. Let $E, E, G$ be Banach spaces, $X \subseteq E$ be compact and $Y \subseteq F$ be 
open. Then, for any integer $n \geq 0$, and $f \in C^{\infty}(X, F)$, define

$$
\|f\|_{n}=\sup \left\{\|f(x)\|+\|D f(x)\|+\ldots+\left\|D^{n} f(x)\right\|: x \in X\right\}<+\infty
$$

and let $C^{n}(X, F)$ denote the Banach space of all $C^{n}$ maps $X \rightarrow F$.

Consider $C^{\infty}(X, F)$ as the intersection of all $C^{n}(X, E), n \geq 0$, and regard it as a locally convex space calibrated by the following sequence of increasing norms defined by (I):

$$
\left\{\|\cdot\|_{n}: n \geq 0\right\} \text {. }
$$

Let $C^{n}(X, Y)$ (respectively $C^{\infty}(X, Y)$ ) be the subset of all $f \in C^{n}(X, F)$ (respectively $f \in C^{\infty}(X, F)$ ) such that $f(X) \subseteq Y$. Then it is clear that $C^{n}(X, Y)$ is open in $C^{n}(X, F)$ for each $n \geq 0$, and $C^{\infty}(X, Y)$ is open in $C^{\infty}(X, F)$ calibrated by (2).

Let $C^{n}(X, G)$ and $C^{\infty}(X, G)$ be similar spaces, where $C^{\infty}(X, G)$ is calibrated by a similar sequence of increasing norms

$$
\left\{\|\cdot\|_{n}: n \geq 0\right\} \text {. }
$$

(2.1) ( $\Gamma$-omega lemma). Let $E, F, G$ be Banach spaces, $X \subseteq E$ compact and $Y \subseteq F$ open. Then, for a fixed $g \in C^{\infty}(Y, G)$, the map

$$
g_{*}: C^{\infty}(X, Y) \subseteq C^{\infty}(X, F) \rightarrow C^{\infty}(X, G): f \rightarrow g_{*}(f)=g \circ f
$$

is $C_{\Gamma}^{\infty}$ with respect to the calibration

$$
\Gamma=\left\{\left(\|\cdot\|_{n},\|\cdot\|_{n}\right): n \geq 0\right\}
$$

for the pair $\left(C^{\infty}(X, F), C^{\infty}(X, G)\right)$ where the first and second $\|\cdot\|_{n}$ are defined by (2) and (3) respectively.

Proof. This follows easily from [12, Remark, p. 26] and [6, Theorem 6, p. 117$]$.

Now let $X$ be a compact $C^{\infty}$ manifold and $\pi: E \rightarrow X, \rho: F \rightarrow X$ be two $C^{\infty}$ (Banach) vector bundles over $X$. Denote by $S^{\infty}(\pi)$ and $S^{\infty}(\rho)$ the spaces of $C^{\infty}$-sections of $\pi$ and $\rho$ respectively. Endow them with the following calibrations. Cover $\pi$ and $\rho$ by a finite number of 
pseudo-compact $V B$-charts $\left(U_{i}, \alpha_{i}^{0}, \alpha_{i}\right)$ and $\left(U_{i}, \alpha_{i}^{0}, \beta_{i}\right), 1 \leq i \leq n$, where $\left\{\left(U_{i}, \alpha_{i}^{0}\right): 1 \leq i \leq n\right\}$ is an atlas of $x \quad[1$, p. 15]. Then each $\gamma \in S^{\infty}(\pi)$ has the following principal part with respect to the $V B$-chart $\left(U_{i}, \alpha_{i}^{0}, \alpha_{i}\right):$

(4) $\quad \tilde{\gamma}_{\alpha_{i}}: \alpha_{i}^{0}\left(\bar{U}_{i}\right) \rightarrow E_{\alpha_{i}}\left(1 \leq i \leq n, \bar{U}_{i}\right.$ being the closure of $\left.U_{i}\right)$ with $\tilde{\gamma}_{\alpha_{i}} \in C^{\infty}\left(\alpha_{i}^{0}\left(\bar{U}_{i}\right), E_{\alpha_{i}}\right)$ and $\alpha_{i}^{0}\left(\bar{U}_{i}\right)$ is compact.

For each $r \geq 0$, define

(5) $\left\|\gamma_{\alpha_{i}}\right\|_{r}=\sup \left\{\left\|\tilde{\gamma}_{\alpha_{i}}(x)\right\|+\left\|D \tilde{\gamma}_{\alpha_{i}}(x)\right\|+\ldots+\left\|D^{r} \tilde{\gamma}_{\alpha_{i}}(x)\right\|: x \in \alpha_{i}^{0}\left(\bar{U}_{i}\right)\right\}$ and, for each $\gamma \in S^{\infty}(\pi)$, define

$$
\|\gamma\|_{r}=\sum_{i=1}^{n}\left\|\tilde{\gamma}_{\alpha_{i}}\right\|_{r}
$$

Then endow $S^{\infty}(\pi)$ with the following calibration

$$
\Gamma_{S^{\infty}(\pi)}=\left\{\|\cdot\|_{r}: r \geq 0\right\} \text {. }
$$

Similarly, $S^{\infty}(\rho)$ is endowed with the calibration

$$
\Gamma_{S^{\infty}(p)}=\left\{\|\cdot\|_{r}: r \geq 0\right\} \text {. }
$$

Now, if $\Omega \subseteq E$ is an open set such that $\pi / \Omega: \Omega \rightarrow X$ is surjective, let $S^{\infty}(\Omega) \subseteq S^{\infty}(\pi)$ be the open set of sections with image contained in $\Omega$.

If $f: \Omega \subseteq E \rightarrow F$ is a $C^{\infty}$ fibre-preserving map, let

$$
f_{*}: S^{\infty}(\Omega) \subseteq S^{\infty}(\pi) \rightarrow S^{\infty}(p)
$$

be the induced map defined by $f_{*}(\gamma)=f 0^{\circ} \gamma$ for all $\gamma \in S^{\infty}(\Omega)$. Then the local $\Gamma$-omega lemma in (2.1) can be globalised as follows.

$$
\text { (2.2) Let } \pi: E \rightarrow X, \rho: F \rightarrow X, f: \Omega \subseteq E \rightarrow F \text { be as above. }
$$

Then $f_{*}: S^{\infty}(\Omega) \subseteq S^{\infty}(\pi) \rightarrow S^{\infty}(\rho)$ is $C_{\Gamma}^{\infty}$ with respect to the calibration $\Gamma=\left\{\left(\|\cdot\|_{r},\|\cdot\|_{r}\right): r \geq 0\right\}$ given by (7) and (8) for the pair 
$\left(S^{\infty}(\pi), S^{\infty}(\rho)\right)$, and, for any integer $r \geq 0$, the rth $\Gamma$-derivative of $f_{*}$ is given by

$$
D^{r} f_{*}=\left(\partial^{r} f\right)_{*}
$$

where $\partial$ denotes the vertical derivative [1].

Proof. Cover $\pi$ and $\rho$ by a finite number of pseudo-compact $V B$-charts as above. Apply (2.1) to the composite of the second projection and the local representative of $f$, and then use the definition of the vertical derivative.

Now, back to the space $C^{\infty}(X, Y)$. Let $s: T Y \rightarrow T^{2} Y$ be a $C^{\infty}$ spray on $Y$. Then there is an open neighbourhood $D_{\mathcal{S}} \subseteq T Y$ of the zero-section and an open neighbourhood $F_{S} \subseteq Y \times Y$ of the diagonal such that $\operatorname{Exp}^{s}: D_{s} \rightarrow F_{s}$ is a $C^{\infty}$-diffeomorphism [1]. If $f \in C^{\infty}(X, Y)$ we have the diffeomorphism $s_{f} \equiv f^{*} \operatorname{Exp}^{s}: f^{*} D_{s}+D_{f, s}$ where $D_{f, s} \subseteq X \times Y$ is an open neighbourhood of the graph of $f$.

If $U_{f, s} \subseteq C^{\infty}(X, Y)$ consists of maps $g$ such that $\operatorname{graph}(g) \subseteq D_{f, s}$, then the map

$$
\phi_{f, s}: U_{f, s} \rightarrow C_{f}^{\infty}(X, T Y) \equiv S^{\infty}\left(f^{*} T Y\right)
$$

defined by $\phi_{f, s}(g)=s_{f}^{-1} \circ \operatorname{graph}(g)$ is a homeomorphism of $U_{f, s}$ onto an open subset of $C_{f}^{\infty}(X, T Y)$, the space of $C^{\infty}$ vector-fields along $f$. We call the pair $\left(U_{f, s}, \phi_{f, s}\right)$ a natural chart for $C^{\infty}(X, Y)$.

(2.3) Let $X$ be a compact $C^{\infty}$ manifold and $Y$ be a finitedimensional $C^{\infty}$ manifold. Then the family $\left(U_{f, s}, \phi_{f, s}\right)$ of natural charts is a $\Gamma$-atlas of class $C^{\infty}$ on $C^{\infty}(X, Y)$ if, for each $f \in C^{\infty}(X, Y)$, we take as calibration for $S^{\infty}\left(f^{*} T Y\right)$ the one defined by (7). Hence $C^{\infty}(X, Y)$ is a $\Gamma$-manifold of class $C^{\infty}$ modelled on the $\Gamma$-family $\left\{S^{\infty}\left(f^{*} T Y\right): f \in C^{\infty}(X, Y)\right\}$.

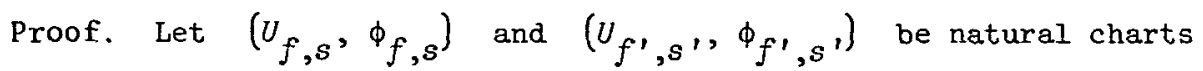
and suppose that $U_{f, s} \equiv U_{f^{\prime}, s^{\prime}}$. It suffices to show that $\phi_{f^{\prime}, s^{\prime}} \circ \phi_{f, s}^{-1}$ 
is a $C_{\Gamma}^{\infty}$-diffeomorphism.

But it is clear that $\phi_{f^{\prime}, s^{\prime}} \circ \phi_{f, s}^{-1}=F_{*}$ where

$$
F=\left[f^{\prime *} \operatorname{Exp}^{s}\right]^{-1} \circ\left[f^{*} \operatorname{Exp}^{s}\right] \text {. }
$$

Since $s, s^{\prime}$ are $C^{\infty}$ sprays and $f, f^{\prime}$ are $C^{\infty}$, it is clear that $F$ is a $C^{\infty}$ fibre-preserving map. Thus, by (2.2), $F_{*}$ is $C_{\Gamma}^{\infty} \cdot$ Clearly, $F_{*}^{-1}=\left(F^{-1}\right)_{*}$, so $F_{*}$ is a $C_{\Gamma}^{\infty}$-diffeomorphism.

As an immediate consequence, the space $\operatorname{Diff}^{\infty}(X)$ of all $C^{\infty}$-diffeomorphisms of a compact $C^{\infty}$ manifold $X$ is a $\Gamma$-manifold of class $C^{\infty}$. Similarly, the space $\operatorname{Emb}^{\infty}(X, Y)$ of all $C^{\infty}$-embeddings of $X$ into a finite-dimensional $C^{\infty}$ manifold $Y$ is also a $\Gamma$-manifold of class $C^{\infty}$.

We now give some simple examples of $B \Gamma$-manifolds of class $C^{\infty}$. Let $X$ be a compact $C^{\infty}$ manifold as always, and let $Y$ be either the cylinder, or the cone, or the l-sphere in $\mathbb{R}^{3}$ defined in $[5$, pp. 115-117]. Then we have explicit formulae for the corresponding exponential maps [5, pp. 116-118] and, using these formulae, it can be seen that $C^{\infty}(X, Y)$ is a $B \Gamma$-manifold of class $C^{\infty}[10]$.

More generally, let us denote by $\mathcal{C}$ the family of all Riemannian manifolds $Y$ such that $C^{\infty}(X, Y)$ can be given a $B \Gamma$-manifold structure. Then it can be seen that

(i) every Euclidean space $\mathbb{R}^{n}$ belongs to $\mathcal{C}$,

(ii) if $Y \in \mathcal{C}$ and $Z \in \mathcal{C}$, then the product $Y \times Z \in \mathcal{C}$,

(iii) if $Y \in \mathcal{C}$ and $Z$ is isometric to $Y$, then $Z \in \mathcal{C}$.

In particular, all flat manifolds belong to $\mathcal{C}$. The answer to the problem of whether $C$ contains a non flat manifold is still not known.

\section{The Smale Density Theorem}

In this section, for the sake of generality and the possibility of application, we state and prove the Smale Density Theorem [2] in its general form which is due to Yamamuro (see [10]). The $B \Gamma$-version (5.1) in $[11$, p. 336] then follows immediately. 
Let $F$ be a $\Gamma$-family, $X, Y$ be $\Gamma$-manifolds of class $C^{r}(r \geq 1)$ modelled on $E, F \in F$ respectively. Let $f: X \rightarrow Y$ be a mapping and $x \in X$ be a point. Then a pair of $\Gamma$-charts of class $c^{r},(U, \alpha)$ and $(V, \beta)$, of $X$ and $Y$ respectively is said to be a pair of strong $\Gamma$-charts of class $c^{r}$ for $f$ at $x$ (or for short, a pair of $S C_{\Gamma}^{r}$-charts for $f$ at $x)$ if and only if $x \in U, f(x) \in V, f(U) \subseteq V$ and the local representative $f_{\alpha \beta}: \alpha(U) \rightarrow \beta(V)$ is a $C_{B \Gamma}^{r}$ map. We say that $f$ is strongly $C_{\Gamma}^{r}$ at $x$ (for short of class $S C_{\Gamma}^{r}$ at $x$ ) if and only if $f$ is $C_{\Gamma}^{r}$ at $x$, and if in addition there is a pair $\{(U, \alpha),(V, \beta)\}$ of $S C_{\Gamma}^{p}$-charts for $f$ at $x . f$ is a strongly $C_{\Gamma}^{p}$ map (or of class $S C_{\Gamma}^{p}$ ) if and only if it is $S C_{\Gamma}^{p}$ at every $x \in X$. Note that when $X$ and $Y$ are $B \Gamma$-manifolds of class $C^{r}$ then $S C_{\Gamma}^{r}$ maps $X \rightarrow Y$ coincide with $C_{B \Gamma}^{r}$ maps $X+Y$.

Now consider a $S C_{\Gamma}^{r}$-map $f: X \rightarrow Y$ between $\Gamma$-manifolds of class $C^{r}$ $(r \geq 1)$. We say that $f$ has the $B \Gamma$-Fredholm property at $x \in X$ if, with respect to a pair of $S C_{\Gamma}^{r}$-charts $\{(U, \alpha),(V, \beta)\}$, the $\Gamma$-derivative $f_{\alpha \beta}^{\prime}(\alpha(x)): E+F$ is a $B \Gamma$-Fredholm linear map [11], [12]. In this case, we define the index of $f$ at $x$ with respect to the pair of $S C_{\Gamma}^{p}$-charts $\{(U, \alpha),(V, \beta)\}$ by

$$
\operatorname{ind}(f ; x,(U, \alpha),(V, \beta))=\text { ind } f_{\alpha \beta}^{\prime}(\alpha(x))
$$

We say that $f: X \rightarrow Y$ has the $B \Gamma-F r e d h o l m$ property if and only if it has the $B \Gamma-$ Fredholm property at every $x \in X$.

(3.1) Let $X, Y$ be $\Gamma$-manifolds of class $c^{r}(r \geq 1)$ modelzed on $E, F \in F, E$ being sequentially complete, and let $f: X \rightarrow Y$ be a $S C_{\Gamma}^{r}$-map having the $B \Gamma-F r e d h o l m$ property at a point $x \in X$. Then we can always find admissible $\Gamma$-charts of class $C^{2},(U, \alpha)$ and $(V, \beta)$ at $x$ 
and $f(x)$ respectively with the following properties:

(i) $E=E_{1} \oplus_{B \Gamma} E_{2}$ where $E_{1}$ and $E_{2}$ are closed subspaces of $E, \operatorname{dim} E_{1}=n<+\infty, U \subseteq$ domain of $f ; \alpha$ maps $U$ $C_{\Gamma}^{r}$-diffeomorphically onto $B_{1}+B_{2}$ with $B_{i}$ closed, convex, circled neighbourhoods of 0 in $E_{i}(i=1,2)$;

(ii) $F=F_{1} \oplus_{B \Gamma} F_{2}$ where $F_{1}$ and $F_{2}$ are closed subspaces of $F, \operatorname{dim} F_{1}=p<+\infty, f(U) \subseteq V, \beta$ maps $V$

$c_{\Gamma}^{r}$-diffeomorphically onto an open subset of $F_{1} \oplus_{B \Gamma} F_{2}$;

(iii) the Local representative $f_{\alpha \beta}: \alpha(U) \subseteq E \rightarrow \beta(V) \subseteq F$ has the form $f_{\alpha \beta}=n+\Phi \circ P_{2}$, where $n: \alpha(U) \subseteq E \rightarrow F_{1}$ is $c_{B \Gamma}^{p}$ with $\eta^{\prime}(0)=0, \Phi$ is a $B \Gamma$-isomorphism of $E_{2}$ onto $F_{2}$ and $P_{2}$ is the second projection $E=E_{1} \oplus_{B \Gamma} E_{2} \rightarrow E_{2}$.

Proof. Start with a pair of $S C_{\Gamma}^{r}$-charts and proceed as in the proof of [2, Theorem (1.7)] with the use of the Inverse Mapping Theorem [12, Theorem (5.2), p. 45].

(3.2) Let $X, Y$ be as in (3.1) and $f: X \rightarrow Y$ be a $S C_{\Gamma}^{\text {-map }}$ $(r \geq 1)$ having the BГ-Fredholm property. Then $f$ is locally closed.

Proof. This follows quickly from (3.1).

Let $f: X+Y$ be a $C_{r}^{p}$-map $(r \geq 1)$ between $\Gamma$-manifolds of class $c^{r}$. We say that $x \in X$ is a reguzar point of $f$ if and only if the r-tangent map $T_{x} f: T_{x} X \rightarrow T_{f(x)} Y$ is surjective; $x$ is a critical point of $f$ if and only if it is not regular [2]. If $C$ is the set of critical points of $f$, then $f(C) \subseteq Y$ is the set of critical values of $f$ and $Y-f(C)$ is the set of regular values of $f$.

(3.3) Let $X, Y$ be as in (3.1) and (3.2) and let $f: X \rightarrow Y$ be a 
$S C_{\Gamma}^{p}$-map having the $B \Gamma-F r e d h o l m$ property. Then the set of regular points of $f$ is open in $X$, hence the set of critical points of $f$ is closed in $X$.

Proof. This follows from the fact that the set $\mathrm{SL}_{B \Gamma}(E, F)$ of $B \Gamma$-splitting surjections $E \rightarrow F$ is open in $L_{B \Gamma}(E, E)$ [12].

(3.4) ( $\Gamma$-version of Smale Density Theorem). Let $F$ be a $\Gamma$-family, $E, F \in F$ being sequentialzy complete. Let $X, Y$ be $\Gamma$-manifolds of class $c^{r} \quad(r \geq 1)$ modelzed on $E, F$ respectively with $X$ Lindelöf. Let $f: X \rightarrow Y$ be a $S C_{\Gamma}^{r}$-map having the $B \Gamma$-Fredholm property and suppose that, for each $x \in X$, we can find a pair of $S C_{\Gamma}^{r}$-charts $\{(U, \alpha),(V, B)\}$ for $f$ at $x$ such that $r>\max \{0$, ind $(f ; x,(U, \alpha),(V, \beta))\}$. Then the set of regular values of $f$ is residual in $Y$.

Proof. Similar to the proof in [2, p. 43] with the use of (3.1), (3.2) and (3.3).

REMARK. The results in this section still hold if we define the critical points as in $[12$, p. 61].

\section{References}

[1] R. Abraham, Lectures of Smale on differential topology (Columbia University, New York, 1962).

[2] Ralph Abraham, Joel Robbin, Transversal mappings and flows (Benjamin, New York, Amsterdam, 1967).

[3] H.R. Fischer, "Differentialrechnung in lokalkonvexen Räumen und Mannigfaltigkeiten von Abbildungen" (Manuskripte d. Fakultät für Math. und Informatik, Univ. Mannheim, Mannheim [1977]).

[4] Jürg Gutknecht, "Die $C_{\Gamma}^{\infty}$-Struktur auf der Diffeomorphismengruppe einer kompakten Mannigfaltigkeit" (Doctoral Dissertation, Eidgenössische Technische Hochschule, Zürich, 1977). 
[5] D. Gromol, W. KI ingenberg, W. Meyer, Riemannsche Geometrie im Großen (Lecture Notes in Mathematics, 55. Springer-Verlag, Berlin, Heidelberg, New York, 1968).

[6] M.C. Irwin, "On the smoothness of the composition map", Quart. $J$. Math. Oxford Ser. 23 (1972), 113-133.

[7] H.H. Keller, "Differential calculus in Fréchet spaces and manifolds of mappings", preprint.

[8] J.A. Leslie, "On a differential structure for the group of diffeomorphisms", Topology 6 (1967), 263-271.

[9] P. Michor, "Manifolds of smooth maps", Cahiers Topologie Géom. Différentielze 19 (1978), 47-78.

[10] Truong Công Nghê, "Differentiable manifolds modelled on locally convex spaces" (PhD thesis, Australian National University, Canberra, 1977). See also: Abstract, Bull. Austral. Math. Soc. 18 (1978), 303-304.

[11] Truong Công Nghê and S. Yamamuro, "Locally convex spaces, differentiation and manifolds", Comment. Math. Special Issue 2 (1979), 229-338.

[12] Sadayuki Yamamuro, A theory of differentiation in locally convex spaces (Memoirs of the American Mathematical Society, 212. American Mathematical Society, Providence, Rhode Island, 1979).

Department of Pure Mathematics, University of Sydney, Sydney, New South Wales 2006, Australia. 